\title{
Research on Design Reuse System of Parallel Indexing Cam Mechanism Based on Knowledge
}

\author{
Yang Xian-Hai ${ }^{*}$, Meng Lu, Li Qian, Song Lu-Peng and Shang Chun-Xiang
}

School of Mechanical Engineering, Shandong University of Technology, Zibo 255049, China

\begin{abstract}
Design experience using parallel indexing cam mechanism, combined with theoretical knowledge engineering and design reuse, and based knowledge of parallel indexing cam mechanism design reuse systems were researched. Construct a flow chart system, studies the key technology knowledge base, inference engine and other systems to UG for the development platform, the development of a knowledge-based parallel indexing cam mechanism design reuse systems, and through examples of the system can greatly improve the design efficiency.
\end{abstract}

Keywords: Knowledge base, parallel indexing cam mechanism, reasoning mechanism, system development.

\section{INTRODUCTION}

Parallel indexing cam mechanism is shouldering the important mission in the intermittent mechanism. Compared with the traditional indexing mechanism, it has the advantages of high indexing precision, good rigidity, compact structure, easy manufacture etc, which is widely used in various automatic and efficient production machinery of food, light industry, machine tools, packaging, pharmaceutical, tobacco and chemical industry [1-3]. But the parallel indexing cam mechanism is designed by traditional method now. If designers change the organization size, they should design and calculate once again [4]. Therefore, the design reuse system of parallel indexing cam mechanism based on knowledge is developed. It can efficiently use the design knowledge and design results, avoid duplication of labor, shorten the design cycle and improve the design efficiency [5]. The design reuse system of parallel indexing cam mechanism based on knowledge is applied the knowledge engineering, design reuse theory, computer technology and $\mathrm{AD}$ technology in the parallel indexing cam design. The system flow chart is built and the key technologies of knowledge base system and reasoning mechanism system are studied. Under the VC6.0 compiler environment, using UG/Open tools, the system is developed combined with the the Access database.

\section{FLOW CHART'S CONSTRUCTION OF THE DESIGN REUSE SYSTEM OF PARALLEL INDEXING CAM MECHANISM}

Parallel indexing cam mechanism is composed of parallel indexing cam, camshaft, turntable, turntable shaft and roller. In order to realize the reuse of parallel indexing cam mechanism, firstly, realize part design reuse, then assemble, finally realize the mechanism design reuse. Flow chart's construction of the design reuse system of parallel indexing cam mechanism based on knowledge is shown in Fig. (1). According to the design information of parallel indexing cam mechanism, firstly, designer should select the type of institution, and then select the type of parts, and design the parts. The reasoning machine is used to choose the best example in the knowledge base in a certain search rule. If the best example information perfectly matches with the target information, the result is output. If not, through the database management system, the best example is modified in a certain rule, until it fully match with the target information. The result is output and added to the knowledge base in some way. Finally, assemble and 3D model of mechanism is output.

\section{RESEARCH ON THE KEY TECHNOLOGY OF THE DESIGN REUSE SYSTEM OF PARALLEL INDEXING CAM MECHANISM}

\subsection{The Construction of Knowledge Base of the Design Reuse System of Parallel Indexing Cam Mechanism}

To establish the knowledge base of system, firstly, understand the characteristics of the experience knowledge related in the process of the design reuse system of parallel indexing cam mechanism. And then classify and express the experience knowledge. The knowledge base is constructed and managed.

\subsubsection{Knowledge Representation of the Design Reuse System of Parallel Indexing Cam Mechanism}

In system development, the experience knowledge related in the design reuse process of parallel indexing cam has the following characteristics: the decomposition of design reuse task, the complexity of domain knowledge, the variety of expression, the quantity of data knowledge and the fuzzy of experience knowledge. The decomposition of design reuse task is the most important feature of all. Design reuse task of parallel indexing cam mechanism can often be described by a set of relatively independent and different 


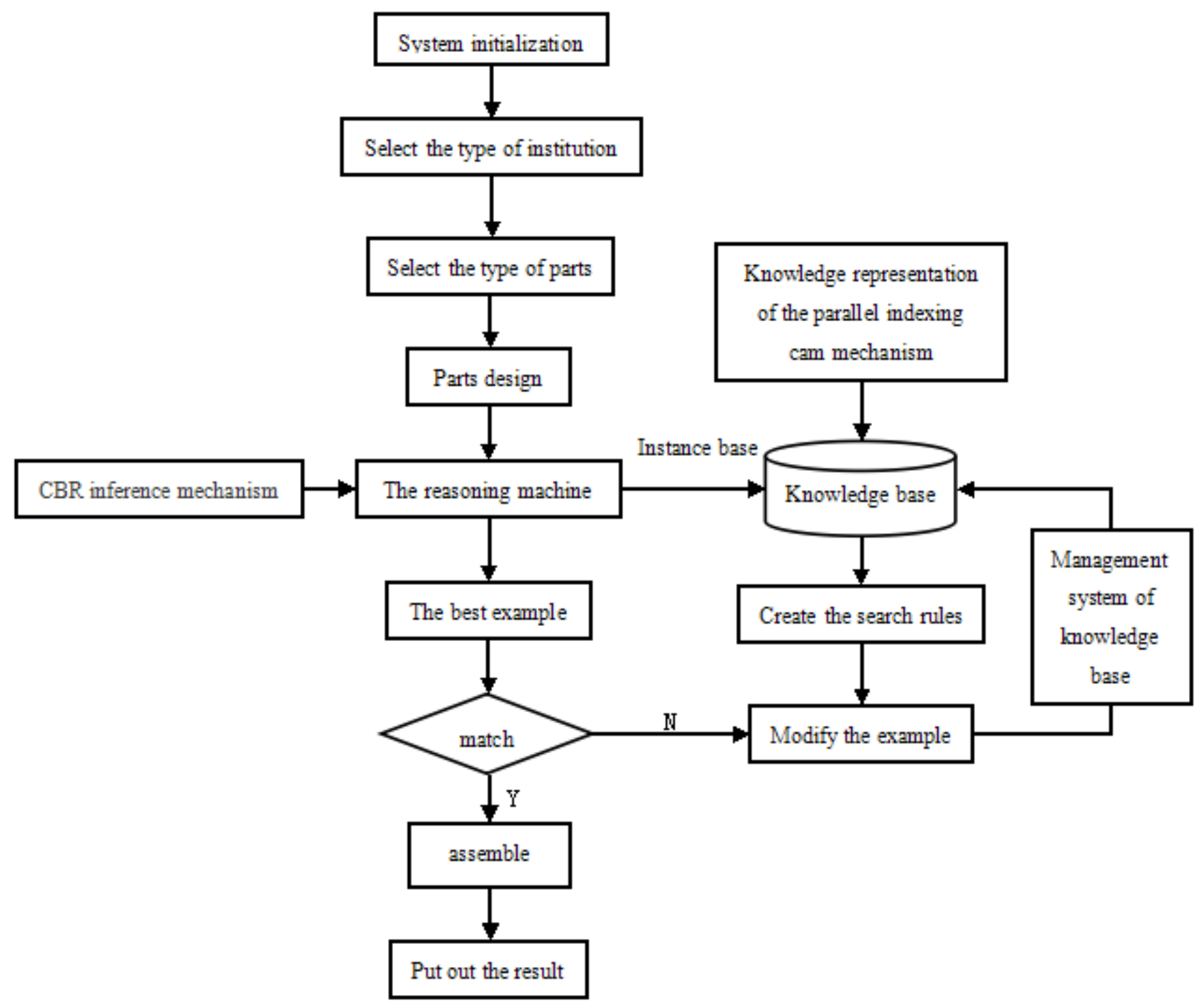

Fig. (1). Flow chart's construction of the design reuse system of parallel indexing cam mechanism.

levels of sub goals. Therefore, the hierarchical model of knowledge base is used to manage the experience knowledge involved in the design reuse process of parallel indexing cam mechanism.

Based on this, design reuse knowledge of parallel indexing cam mechanism is conducted the knowledge representation in different methods. Knowledge existing in the form of framework is used the BNF frame representation. Knowledge existing in the form of formula is used the process representation. Knowledge existing in the form of feature and parameter is used the database representation. Knowledge existing in the form of model is used the database representation and document representation.

\subsubsection{The Structure of the Knowledge Base System}

According to the "reuse of design task decomposition" characteristic of parallel indexing cam design knowledge reuse, the whole design reuse process of parallel indexing cam mechanism is a decision process. Each task can be divided into a plurality of sub goals. Therefore, knowledge module can be divided according to the sub goals. And the modular organization of knowledge base can be used. The structure of the knowledge base system is shown in Fig. (2).

\subsubsection{Management of Knowledge Base System}

The management of knowledge base, specifically, is that the existing knowledge is searched, put in storage, deleted, modified, checked the completeness and redundancy. In this paper, the management of knowledge base is mainly the instance base management and knowledge management.

Instance base management is mainly to store and manage many examples. While database management is mainly for data storage and management. Their features similar, so database management technology can be used to manage instance base.

Let's take external parallel indexing cam mechanism for an example, parallel indexing cam is put in storage by database management technology, as shown in Fig. (3). 


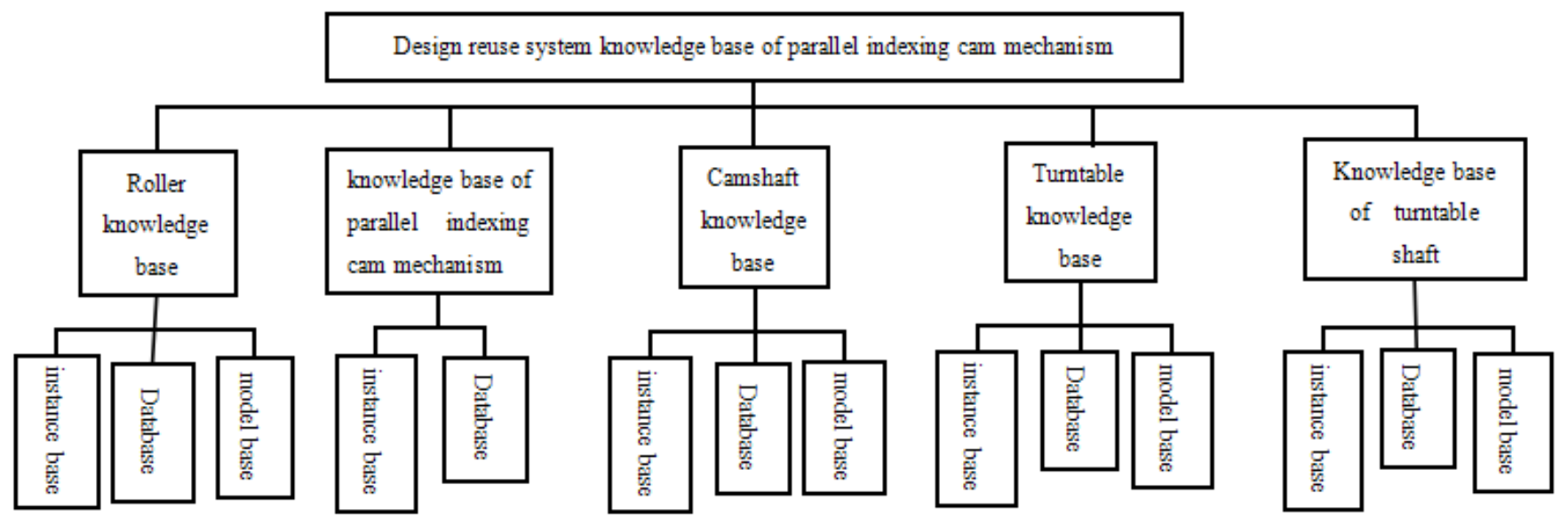

Fig. (2). The structure of the knowledge base system.

\subsection{Reasoning Research of the Design Reuse System of Parallel Indexing Cam Mechanism}

The reasoning machine of the design reuse system of parallel indexing cam mechanism is constructed based on case-based reasoning method. The focus is the case retrieval technique. The case retrieval technique is the important link of the design reuse system of parallel indexing cam mechanism. The precision and accuracy directly affects the reliability of parallel indexing cam mechanism.

\subsubsection{Hierarchical Indexing Model}

The "finished product layer+ parts category layer + instance level" hierarchical index model is constructed parametric and feature and based on the structure of instance base. The model structure is shown in Fig. (4). Finished product layer preserves categorical attributes of parallel indexing cam mechanism. Parts category layer preserves categorical attributes of various parts of parallel indexing cam mechanism. Instance layer can be divided into shape feature, material feature, heat treatment feature, precision feature feature. It can preserve the necessary attributes various examples of parts of parallel indexing cam mechanism, which is used to describe the specific information of parts.

\subsubsection{Case Similarity Algorithm}

The similarity coefficient is a measure of consistent degree between target part and example part. It is shown by $S$. According to the indexing model of parallel indexing cam mechanism, parts similarity is researched in four aspects: shape feature, material feature, heat treatment feature and precision feature. The similarity coefficient formula is as follows:

$S=\sum_{i=1}^{n} S_{i} W_{i}$

In the type: $S$ is the similarity coefficient of target part and some example part; $n$ is the number of overall characteristic example; $i=1,2, \cdots \cdots, n ; W_{j}$ is the weight coefficient of each feature, $W_{i} \in[0,1]$ and $\sum_{i=1}^{n} W_{i}=1$.

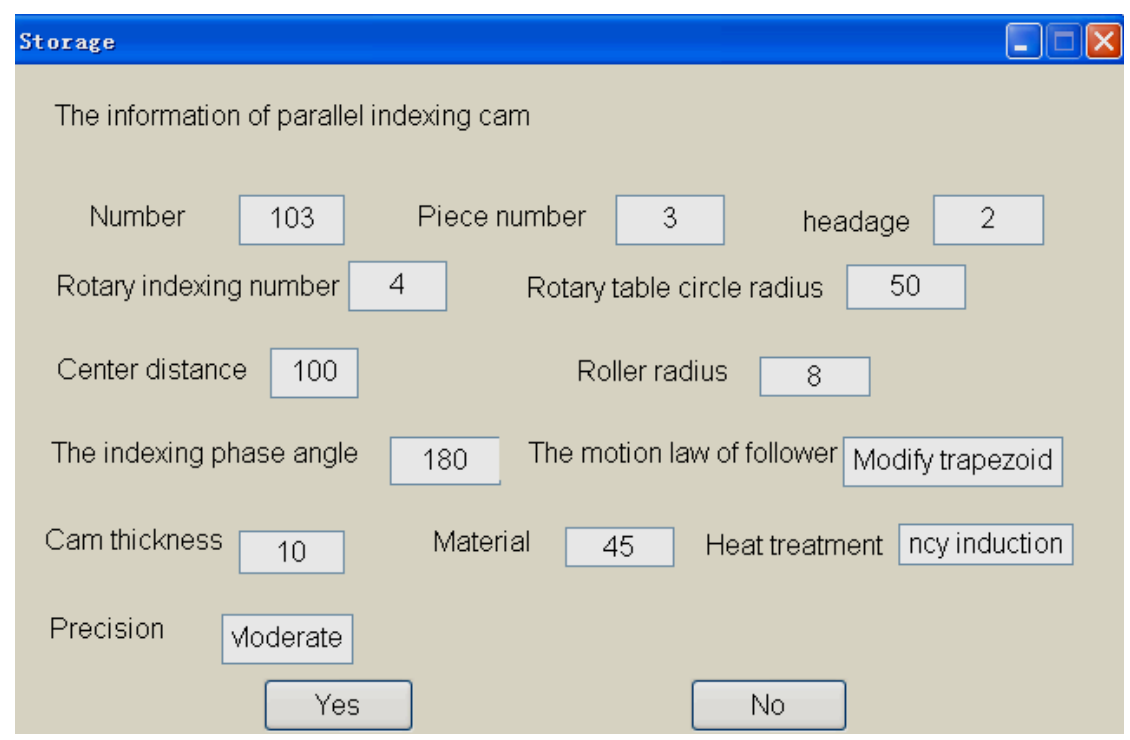

Fig. (3). External storage interface of parallel indexing cam. 


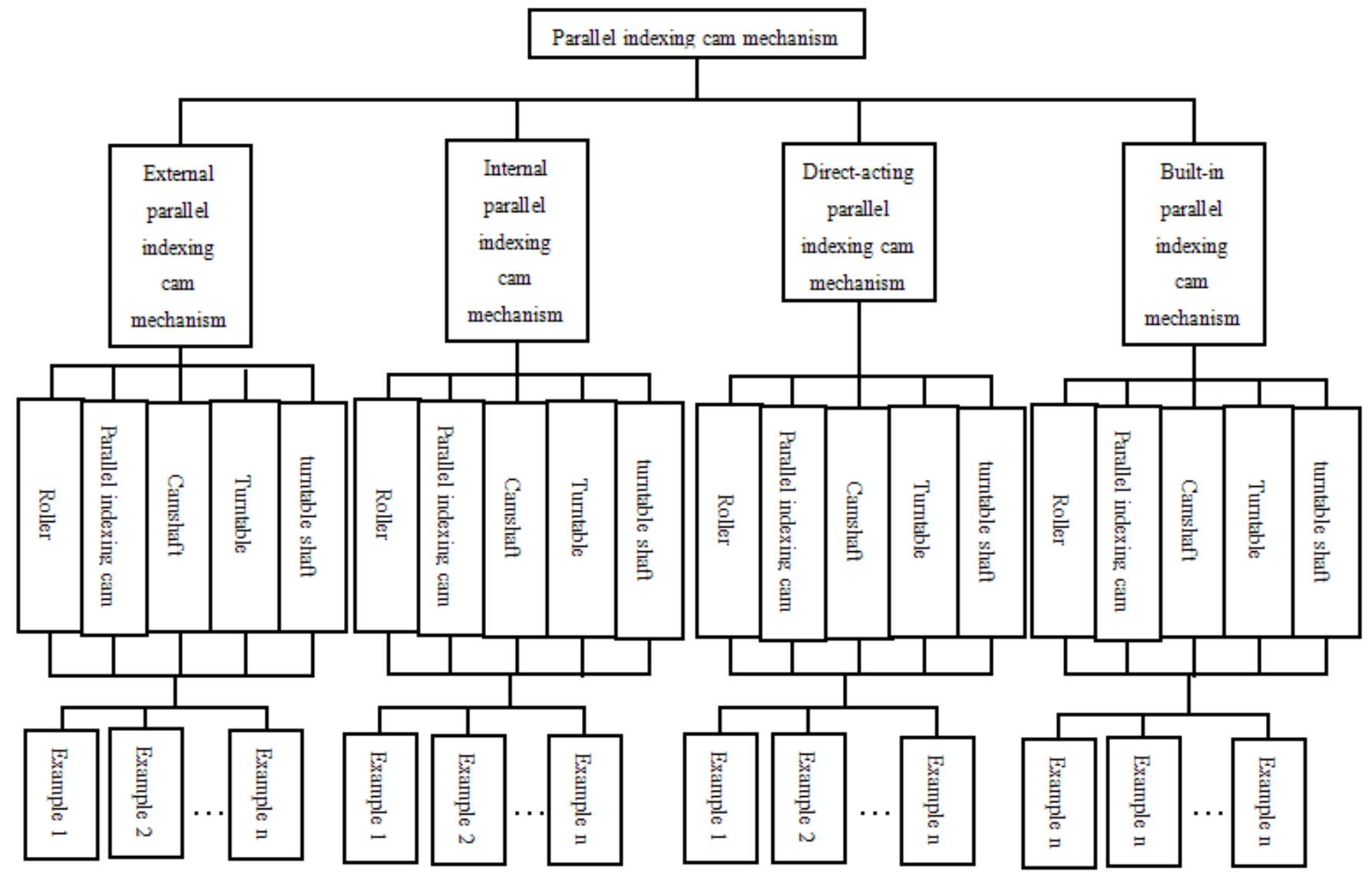

Fig. (4). Hierarchical index model of parallel indexing cam mechanism.

Combined with the experience and knowledge of related field experts and relevant papers, ultimately determine that the shape feature, material feature, heat treatment feature, precision feature weight coefficients are $0.75,0.05,0.1,0.1$, considering the influence of parallel indexing cam mechanism design by the characteristics of the system.

$S_{j}$ is $0 \leq S_{i} \leq 1$. The calculation formula is as follows:

(1) When the characteristic information is the shape feature, set $F_{a}$ as the structure feature number of target part a, $F_{b}$ as the structure feature number of a example part b, $F_{a b}$ as the same structure feature number of two parts. Then

$$
S_{i}=\frac{2 F_{a b}}{F_{\mathrm{a}}+F_{b}}
$$

(2) When the characteristic information is the material feature, heat treatment feature and precision feature, if two messages match, then $S_{i}=1$. If not, $S_{i}=0$. For example, the target part and a example part of the materials are both 45 , then $S_{i}=1$.

\subsubsection{Instance Retrieval Process}

Retrieval strategy of instances include nearest neighbor strategy, inductive reasoning strategy, knowledge guided strategy. The knowledge guided strategy is used in this paper. The realization process is as follows:
(1) According to the type of parallel indexing cam mechanism selected by users, the system judges the subordinate product types of target institutions, namely into the part layer;

(2) According to the target parts information selected by users, system judges the subordinate part type of target part, namely into the instance level;

(3) According to the feature information of the target part, open the corresponding database in accordance with the retrieval strategy;

(4) Match the feature information of all instances and characteristic information of target parts, and calculate the similarity coefficient. Search out instance set which meet the requirements. The similarity coefficient is ranged by size, and select the best example for users to access, such as modification, storage etc..

\section{DEVELOPMENT OF THE DESIGN REUSE SYSTEM OF PARALLEL INDEXING CAM MECHANISM BASED ON KNOWLEDGE}

\subsection{The Design of Design Reuse System}

Environment setting of the design reuse system of parallel indexing cam mechanism based on knowledge is shown in Fig. (5). System development is used UG/Open development tools: UG/Open Manuscript menu, UG/Open UIStyler development UG dialog box. 


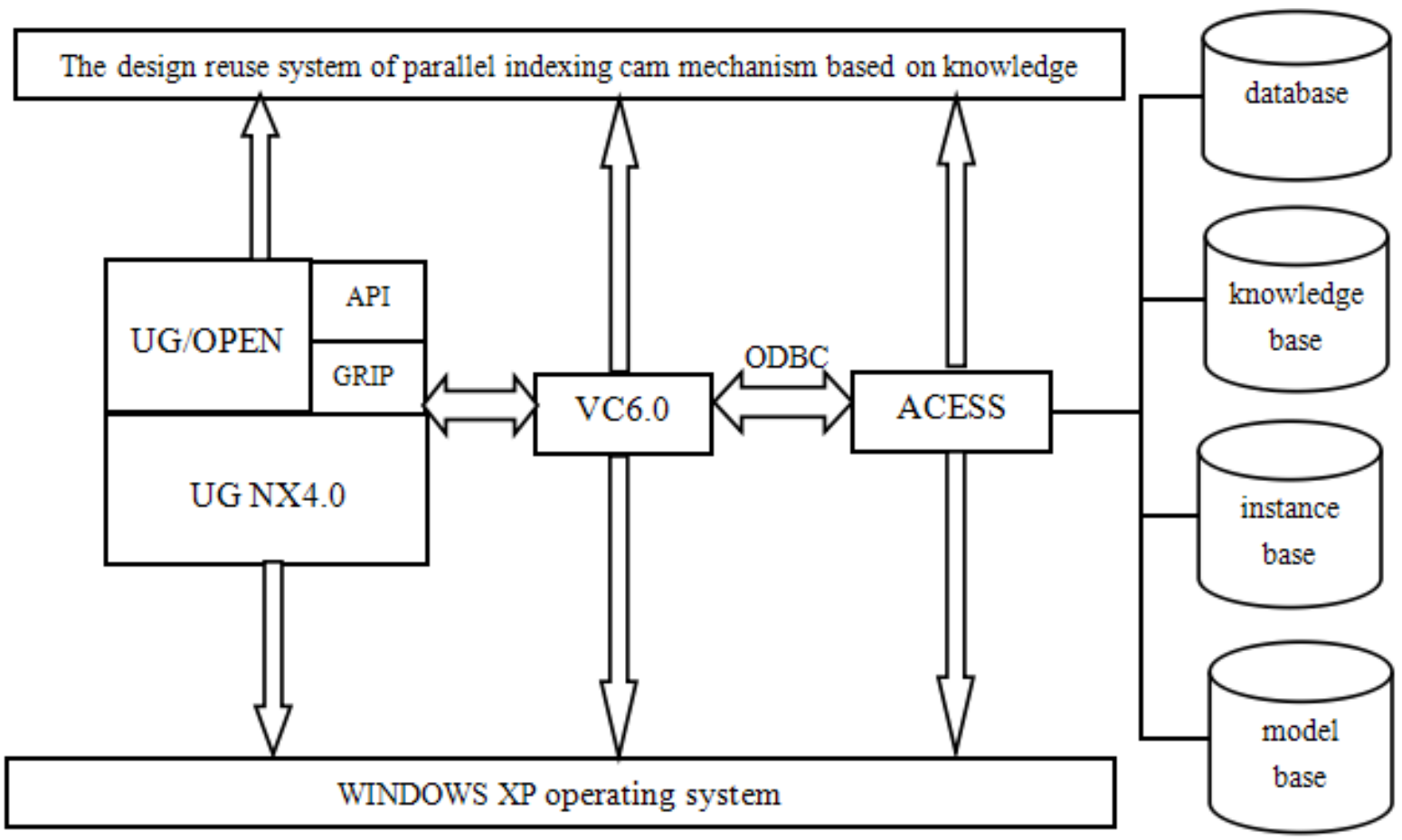

Fig. (5). Design reuse system environment settings.

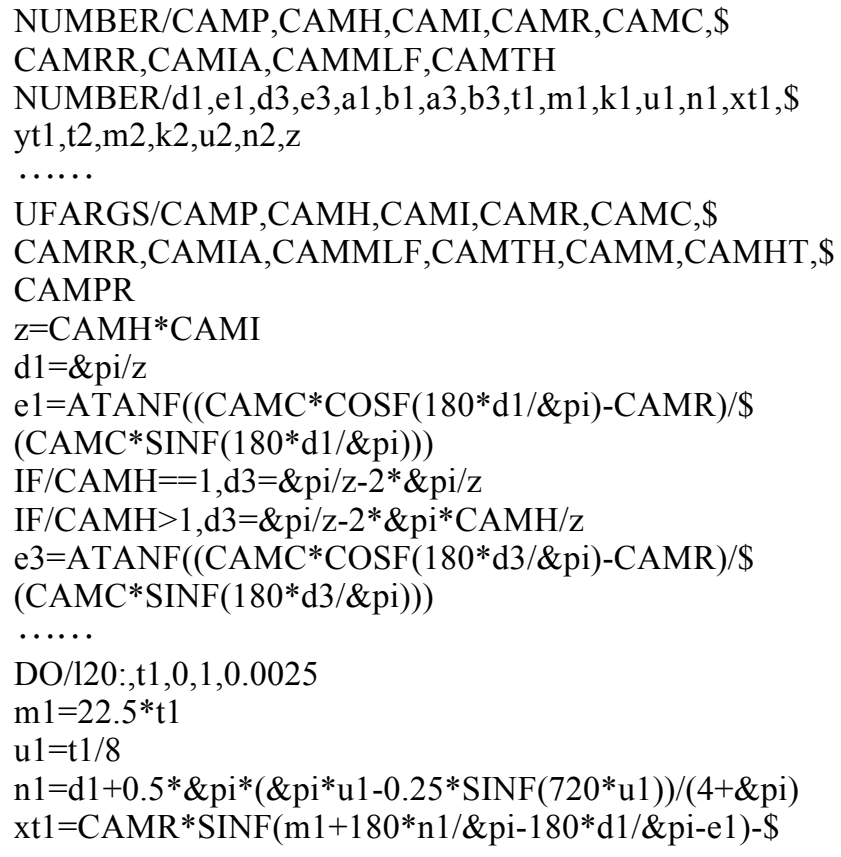

\begin{abstract}
UG/Open API is the interface between UG and external application program. UG/Open Grip is interactive graphical programming language. When the system is developed, because of the complexity of the parallel indexing cam curve, this part is created a template with UG/Open Grip language. The structures of other parts are relatively simple. They can be created templates in the UG platform through the expression and sketch. The following is part of the Grip subroutine code of parallel indexing cam template called by API:
\end{abstract} CAMC*SINF(m1-180*d1/\&pi-e 1$)$ yt $1=-C A M R * \operatorname{COSF}(m 1+180 *$ n1/\&pi-180*d1/\&pi-e 1$)+\$$ CAMC $* \operatorname{COSF}(\mathrm{m} 1-180 * \mathrm{~d} 1 / \& \mathrm{pi}-\mathrm{e} 1)$

$\mathrm{zt} 1=0$

$\mathrm{k} 1=\mathrm{k} 1+1$

$\mathrm{IF} / \mathrm{k} 1<=1000, \mathrm{pt} 1(\mathrm{k} 1)=\mathrm{POINT} / \mathrm{xt} 1, \mathrm{yt} 1, \mathrm{zt} 1$

......

shape(7)=CRVTRM/shape(3),REF,pt(7),FIRST,shape(2),\$

INT,pt(5),NOTRIM,STATUS, 1

shape (8)=SPLINE/APPROX, shape (1..5)

solid=SOLEXT/shape(8),HEIGHT,CAMTH,AXIS, $0,0,1$

.......

ENDIF

stop:

HALT

\subsection{Examples of Design Reuse System}

Taking external parallel indexing cam mechanism for an example, select the mechanism type in the menu, and then select the parts design type. Taking the design of the parallel indexing cam for an example, the knowledge management interface of parallel indexing cam mechanism is appeared, as shown in Fig. (6). Input the design information of parallel indexing cam in the interface, and search. Then select the best example and modify. Return to the design interface of parallel indexing cam, as shown in Fig. (7). 3D model of parallel indexing cam is generated, as shown in Fig. (8). In accordance with the above steps, other parts of parallel indexing cam mechanism are designed. Finally, assemble them. 3D model of parallel indexing cam mechanism is generated, as shown in Fig. (9). 


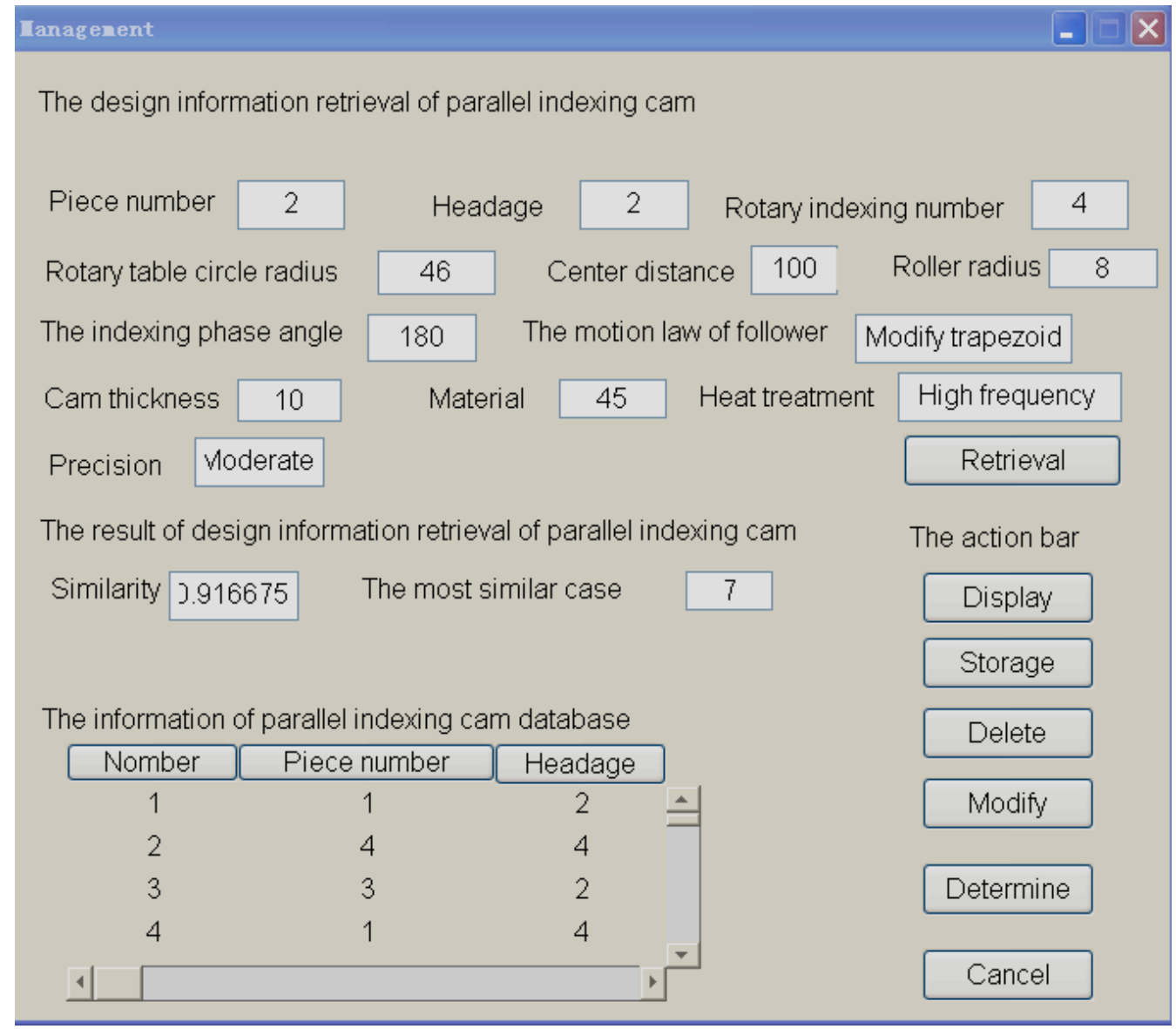

Fig. (6). The knowledge management interface of parallel indexing cam.

\begin{tabular}{|c|c|c|}
\hline Design & & 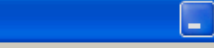 \\
\hline \multicolumn{3}{|c|}{ Knowledge management of parallel indexing cam } \\
\hline \multicolumn{2}{|l|}{ Piece number } & 2 \\
\hline \multicolumn{2}{|l|}{ Headage } & 2 \\
\hline \multicolumn{2}{|l|}{ Rotary indexing number } & 4 \\
\hline \multicolumn{2}{|l|}{ Rotary table circle radius } & 46 \\
\hline \multicolumn{2}{|l|}{ Center distance } & 100 \\
\hline \multicolumn{2}{|l|}{ Roller radius } & 8 \\
\hline \multicolumn{2}{|l|}{ The indexing phase angle } & 180 \\
\hline \multicolumn{2}{|l|}{ The motion law of follower } & Modify trapezoid \\
\hline \multicolumn{2}{|l|}{ Cam thickness } & 10 \\
\hline \multicolumn{2}{|l|}{ Material } & 45 \\
\hline \multicolumn{2}{|l|}{ Heat treatment } & High frequency \\
\hline \multirow[t]{2}{*}{ Precision } & & Moderate \\
\hline & Determine & Cancel \\
\hline
\end{tabular}

Fig. (7). the design dialog of parallel indexing cam. 


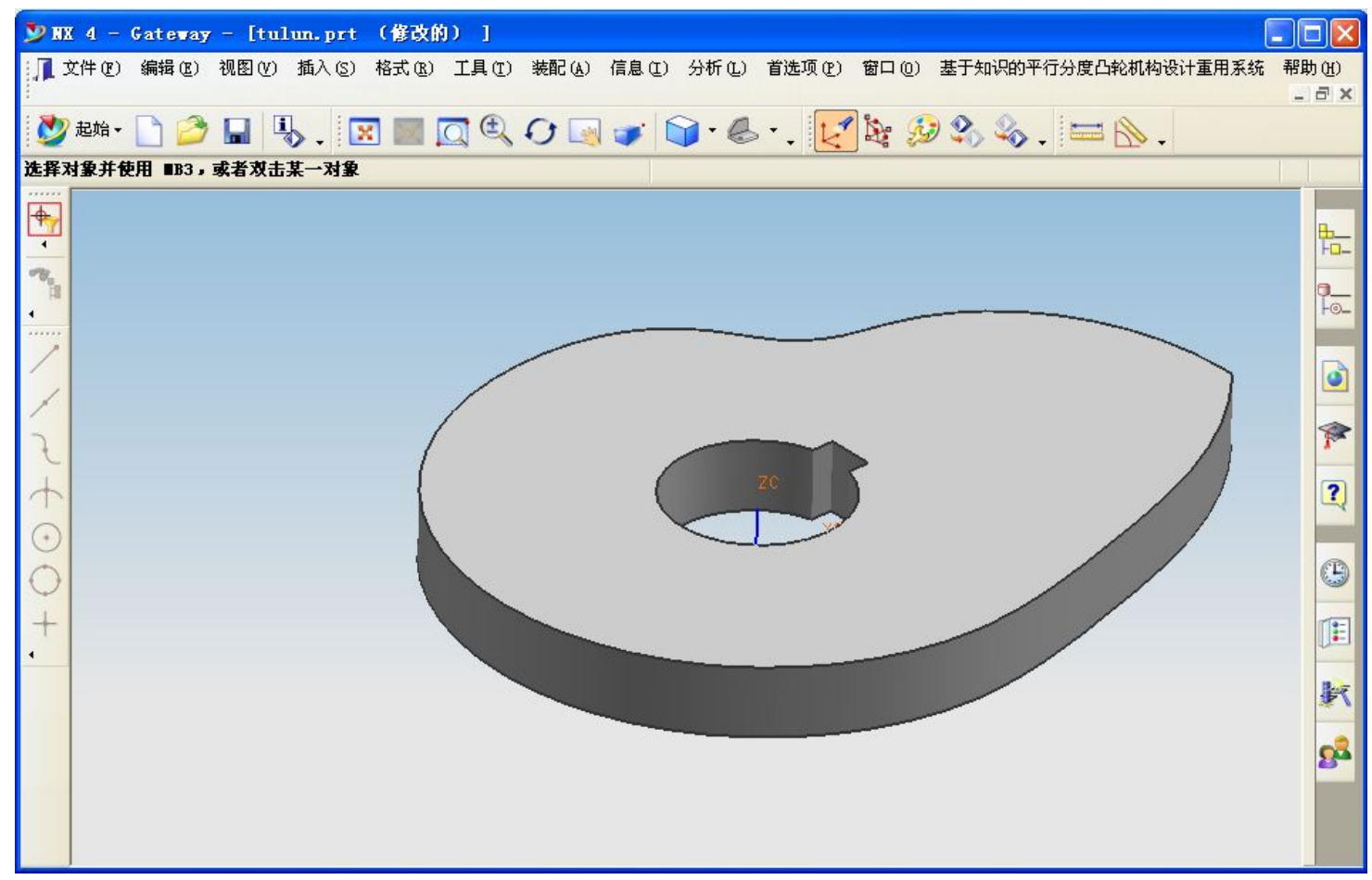

Fig. (8). 3D model of parallel indexing cam.

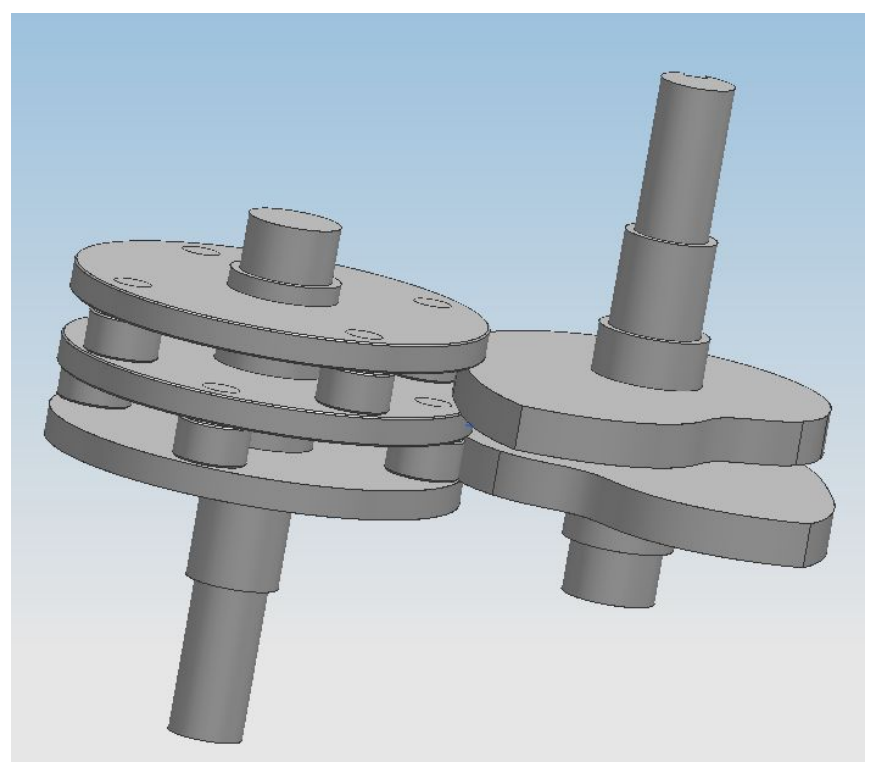

Fig. (9). 3D model of external parallel indexing cam 3D model.

\section{CONCLUSION}

A flow chart of the design reuse system of parallel indexing cam is constructed in this paper. The knowledge base and inference mechanism of design reuse system are focused on. The design reuse system of parallel indexing cam based on knowledge is developed. The external parallel indexing cam as an example runs the system, which realizes the rapid design of the parallel indexing cam, and improves the design efficiency.

\section{CONFLICT OF INTEREST}

The authors confirm that this article content has no conflict of interest.

\section{ACKNOWLEDGEMENTS}

This paper be Supported of Natural Science Foundation of Shandong Province, No. ZR2013EEM011 \& ZR2014EEM046.

\section{REFERENCES}

[1] H. Zou, H. Yin, Design and Application Innovation Of The Intermittent Motion Mechanism, Mechanical Engineering Press, 2008.

[2] X. Liu, Y. Wang, X. Yang, "Research and development on CAD/CAM system of parallel indexing cam," J. Shandong Univ. Technol, vol. 25, no. 5, pp. 75-78, 2011.

[3] W. Hu, X. Yang, L. Meng, "Research on intelligent design system for parallel indexing cam mechanism based on knowledge", Manuf., Automat., vol. 25, no. 5, pp. 75-78, 2011.

[4] W. Du, The Case-Based Reasoning Design and Realization of Gear Reducer 3D CAD Design System, University of Electronic Science and Technology, 2012.

[5] Z. Huang, Research on Integrated System for Injection Mold Design Based on Knowledge, Huazhong University of Science and Technology, China, 2009. 